\title{
Johann Jakob Wepfers experimentelle Toxikologie *
}

Von Andreas-Holger Maehle

Im Jahr 1679 erschien in Basel die toxikologische Schrift « Cicutae aquaticae historia et noxae. Commentario illustrata», verfaßt von dem Schaffhauser Stadtarzt Johann Jakob Wepfer. ${ }^{1}$ Mit diesem Werk begründete Wepfer als Vertreter einer empirisch-naturwissenschaftlich orientierten Medizin die Methodik der systematischen, tierexperimentellen Giftprüfung.

\section{Problem und Anlaß}

Den Ausgangspunkt seiner Untersuchungen bildeten mehrere Vergiftungsfälle durch den Wasserschierling (Cicuta aquatica, Cicuta virosa L.). Im März 1670 hatten in dem badischen Dorf Almanshofen acht Kinder vom Wurzelstock dieses Doldenblütlers gegessen. Schwere epileptiforme Krampfanfälle waren die Folge, in zwei Fällen führte die Vergiftung zum Tode. ${ }^{2}$ Wepfer wunderte sich, daß das Gift des Wasserschierlings Krämpfe erregte, schien doch diese Symptomatik der klassischen Beschreibung einer Schierlingsvergiftung, nämlich der vom Tod des Sokrates in Platos «Phaidon », zu widersprechen. Sokrates starb unter dem Bild einer aufsteigenden Lähmung. Handelte es sich nun um ein « warmes», « erhitzendes», d. h. krampferregendes Gift oder um ein «kältendes», d.h. lähmendes? ${ }^{3}$

Der Grund für die vermeintliche Diskrepanz lag aber nicht auf pharmakologisch-toxikologischem Gebiet, sondern - wie wir heute wissen - auf botanischem. Zu Wepfers Zeit unterschied die Pflanzenkunde im Allgemeinen noch nicht scharf zwischen dem Wasserschierling (Cicuta virosa L.) und dem gefleckten oder echten Schierling (Conium maculatum L.). ${ }^{4}$ Das Cicutoxin des Wasserschierlings ist ein zentral angreifendes Krampfgift, wie R. Boehm rund zweihundert Jahre nach Wepfer in seiner Studie «Über den

* Dieser Beitrag ist eine Zusammenfassung der Inaugural-Dissertation des Verfassers «Johann Jakob Wepfer (1620-1695) als Toxikologe», Bonn 1983, die unter der Betreuung von Herrn Prof. Dr.med. Nikolaus Mani, Medizinhistorisches Institut der Universität Bonn, entstand. Herrn Professor Mani sei an dieser Stelle für Anregung und Kritik auch zu vorliegender Arbeit herzlich gedankt.

Gesnerus 1/2 (1985) 
giftigen Bestandtheil des Wasserschierlings (Cicuta virosa) und seine Wirkungen» zeigte. ${ }^{5}$ Der gefleckte Schierling enthält das Alkaloid Coniin, das durch vorwiegende Wirkung auf Rückenmark und Medulla oblongata nach einer kurzen Erregungsphase eine Lähmung der motorischen und bulbären Zentren in aufsteigender Reihenfolge hervorruft. ${ }^{6}$ Es ist sehr wahrscheinlich, daß im Giftbecher des Sokrates Conium maculatum enthalten war. ${ }^{7}$

Für Wepfer hingegen war der erwähnte Widerspruch ebenso unlösbar wie beunruhigend, denn hier stellte offenkundig die klinische Beobachtung die Autorität der klassischen Literatur in Frage. Es galt, die Evidenz der eigenen Erfahrung gegen das tradierte Wissen zu verteidigen, ${ }^{8}$ denn auch die meisten medizinischen Autoren der Vergangenheit hatten den Schierling als «kältendes» Gift klassifiziert. ${ }^{9}$ Zu diesem Zweck unternahm Wepfer eine größere Zahl von Vergiftungsversuchen an verschiedenen Tieren. ${ }^{10} \mathrm{Er}$ experimentierte mit Wasserschierling und einer Reihe anderer Pflanzengifte, die nach allgemeiner Auffassung eine «warme» und «erhitzende» Qualität besitzen sollten: Blauer Eisenhut (Aconitum napellus L.), Kokkelskörner (Cocculi indici von Menispermum cocculus L.), Brechnuß (Nux vomica von Strychnos nux-vomica L.), weiße Nieswurz oder weißer Germer (Veratrum album L.), Jalape (Exogonium purga Bentham) und bittere Mandeln (Prunus amygdalus Batsch. var. amara Focke). Wepfer verglich diese in ihrer Vergiftungssymptomatik und den pathologisch-anatomischen Veränderungen, die sie am Versuchstier hinterließen, mit dem Wasserschierling. Unter diesen Versuchen findet sich auch einer mit dem Erdschierling (Cicuta terrestris), bei dem es sich vermutlich um Conium maculatum handelte. ${ }^{11}$ Da Wepfer hier aber nur ein uncharakteristisches Vergiftungsbild beobachten konnte, kam es nicht zu möglichen Schlußfolgerungen bezüglich des oben dargestellten Problems. Schließlich wurden von Wepfer Antimonpräparate (Antimonblüte, Metallsafran, Spießglanzglas) und Quecksilbermittel (Kalomel, Sublimat, metallisches Quecksilber) tierexperimentell auf ihre Toxizität hin untersucht. Die weitgehend übereinstimmende Symptomatik nach Gabe der genannten pflanzlichen Gifte mit dem Bild der Wasserschierlingsvergiftung (Erbrechen, Diarrhoe, Krämpfe) bestärkte ihn in der Annahme, daß das Gift der Cicuta aquatica als reizendes, scharfes und «erhitzendes» zu charakterisieren sei. Über diese allgemeine Kennzeichnung hinaus entwickelte Wepfer in der Diskussion seiner Tierversuche eine kohärente Theorie über den Pathomechanismus der Wasserschierlingsvergiftung. 


\section{Wepfers Methodik}

Als Versuchstiere dienten Wepfer meist Hunde, ferner prüfte er die Gifte an Wolf, Katze, Taube, Storch und einem Mäusebussard. Wepfer verabreichte die giftige Substanz peroral. Eigene Experimente mit intravenösen Injektionen beschreibt er nicht. Letzteres ist insofern erstaunlich, als etwa um die gleiche Zeit Johann Sigismund Elsholtz, Leibarzt des Kurfürsten zu Brandenburg, und Johann Jakob Harder, Professor der Anatomie in Basel, Berichte über i.v. Injektionen mineralischer und pflanzlicher Gifte im Tierversuch veröffentlicht haben und Wepfer deren Versuche auch kannte. ${ }^{12}$ H.Buess (1946) vermutete als Ursache für die Zurückhaltung Wepfers gegenüber der damals neuen Methode dessen konservative, vorsichtig abwägende und nüchterne Einstellung als Forscher. ${ }^{13}$

Der Ablauf der toxikologischen Experimente wurde von Wepfer in Versuchsprotokollen («Historiae») festgehalten. Nach einer kurzen Beschreibung des Versuchstieres werden Art und Dosis des Giftes sowie der Zeitpunkt der peroralen Applikation angegeben. Es folgt das Protokoll über die Vergiftungssymptome unter Berücksichtigung ihrer Reihenfolge, Intensität und Dauer. Wenn die Symptomatik anzeigt, daß der Tod des Tieres bevorsteht, wird meist viviseziert. Bei einigen Versuchen wird die Sektion unmittelbar post mortem ausgeführt. Zunächst wird der Bauchraum eröffnet. Unter Vivisektionsbedingungen beschreibt Wepfer Magen und Därme insbesondere bezüglich Dehnungszustand und Peristaltik sowie die Zwerchfellbewegungen bei Singultus, Erbrechen, Inspiration und Exspiration. Im Zuge der weiteren Sektion werden Magen- und Darminhalt inspiziert und nach deren Entfernung die Schleimhäute auf entzündliche Veränderungen und Erosionen untersucht. Oft wird der Ductus thoracicus freigelegt und besichtigt, ebenso die Cisterna chyli und die größeren mesenterialen Lymphgefäße. Nach Öffnung des Brustraumes und Inspektion der Thorakalorgane werden die Herzkammern und die großen Gefäße aufgeschnitten. Das austretende Blut beurteilt Wepfer nach Farbe, Konsistenz und Gerinnselbildung.

Der Stellenwert eines methodischen Vorgehens beim toxikologischen Tierversuch wurde von Wepfer dadurch betont, daß er im Vorwort seiner Abhandlung über den Wasserschierling eine kurze Versuchsanleitung für Kollegen, die Gifte studieren wollen, gab. Der Text lautet ins Deutsche übersetzt wie folgt:

«Wenn die Kollegen auf diesem Gebiet (sc. der Vergiftungen) nicht 
weiterkommen sollten, werden sie ganz besonders mit folgender Methode nach der Wahrheit forschen können, und zwar ohne großen Aufwand oder ängstliche Besorgnis: Häufig verwendet man Hunde, die als Märtyrer der Anatomen gelten, zur Vivisektion, um die Bewegungen des Magens, der Därme, des Zwerchfells, des Herzens, die chylusführenden Lymphgefäße, das Receptaculum ( = Cisterna) chyli, den Ductus thoracicus und ähnliches zu sehen. In ein und demselben Experiment werden sie einem Hund vor der Sektion etwas von diesen (sc. Giften) eingeben können und, indem sie sich beide Wünsche zugleich erfüllen, auch oft das, was sie möchten, klarer sehen: Zum Beispiel möge ein Versuch mit der Brechnu $\beta$ gemacht werden. Zuerst soll jene als Ganzes gegeben werden, in welcher Form sie, wie manche behaupten, keine Krämpfe hervorrufen soll, sodann in Stückchen geschnitten oder geraspelt; oder es möge deren Essenz eingeflößt werden oder, wenn man in Erwägung zieht, daß das Experiment wegen des Weingeistes unzuverlässig wird, soll deren Extrakt in einer Brühe gelöst verabreicht werden ... Das möge einem recht alten, mittelalten oder säugenden Hund auf leeren oder vollen Magen gegeben werden. Aufmerksam sollen Zahl, Reihenfolge und Stärke der Symptome beobachtet werden. Der Hund soll lebend oder sofort nach Todeseintritt seziert werden. Zu betrachten sind das Innere der Speiseröhre, des Magens und der Därme, die größeren Blutgefäße sollen aufgeschnitten werden, solange der Hund noch warm ist. So wird mit Sicherheit vieles klarer werden. ${ }^{14}$

Die Diskussion der Experimente setzte Wepfer deutlich vom objektiven Versuchs- und Sektionsprotokoll ab. In sogenannten «Scholia» erörtert er die Wertigkeit der erhobenen Befunde und ordnet diese in ein hypothetisches Konzept über den Wirkungsmechanismus des untersuchten Giftes ein. Aussagen und Ergebnisse anderer Autoren werden diskutiert. Besonders die experimentellen Arbeiten von Francesco Redi und Moyse Charas über das Viperngift sind für ihn vorbildlich. ${ }^{15}$ Es ist zu beachten, daß Wepfer trotz genauer Dosisangeben noch keine quantitativ-vergleichenden Untersuchungen anstellte. Seine Experimente sind auf die qualitative Charakterisierung der jeweiligen Giftwirkung ausgerichtet.

\section{Die wichtigsten Befunde}

Wepfer beschrieb mehrfach die generalisierten, tonisch-klonischen, epileptiformen Krampfanfälle bei Wasserschierlings- und Kokkelskornvergiftung 
sowie die Starrkrämpfe nach Vergiftung mit Brechnüssen. Wir finden hier geradezu klassische Beschreibungen der Cicutoxin-, Pikrotoxin- und Strychninvergiftung. Bei seinen Versuchen mit bitteren Mandeln konnte Wepfer Respirationsstörungen und Erstickungskrämpfe beobachten (Bild der akuten Blausäurevergiftung). Den hier typischen Sektionsbefund, das hellrote und flüssige Leichenblut, stellte er wiederholt heraus. Wepfers toxikologische Prüfung damals gebräuchlicher Emetica und Purgantien zeigte ihm deren Schadwirkung auf den Magendarmkanal an. Nach Gabe des laxierenden Magisterium Jalapae (aus Jalapenharz), ebenso nach emetischen Antimonpräparaten, sah er Entzündungen der Magen- und Darmschleimhaut, zusätzliche Erosionen und Nekrosen nach peroraler Applikation von Sublimat. Eine Übersicht aller wesentlichen experimentell-toxikologischen Befunde Wepfers ${ }^{16}$ aus «Cicutae aquaticae historia et noxae» zeigt die Tabelle.

\section{J. J. Wepfers experimentelle Befunde aus «Cicutae aquaticae historia et noxae", Basel 1679}

\begin{tabular}{|c|c|c|}
\hline Gift & Versuchstiere & pathologische Befunde \\
\hline $\begin{array}{l}\text { Wasserschierling } \\
\text { (Cicuta virosa L.) }\end{array}$ & $\begin{array}{l}2 \text { Hunde, } \\
2 \text { Wölfe, } \\
1 \text { Mäusebussard }\end{array}$ & $\begin{array}{l}\text { Erbrechen; Salivation; Singultus; } \\
\text { Diarrhoe; gesteigerte Diurese; } \\
\text { taumelnde Körperbewegungen; } \\
\text { generalisierte, tonisch-klonische, } \\
\text { epileptiforme Krampfanfälle mit } \\
\text { initialem Aufschrei; Respirations- } \\
\text { störungen bis zur finalen Atem- } \\
\text { lähmung. } \\
\text { Sektion: geblähter Magen; } \\
\text { entzündliche Rötung der Magen- } \\
\text { und Darmschleimhaut. }\end{array}$ \\
\hline $\begin{array}{l}\text { Erdschierling } \\
\text { (Conium maculatum L.) }\end{array}$ & 1 Hund & $\begin{array}{l}\text { Brechreiz; Diarrhoe; taumelnde } \\
\text { Körperbewegungen; Schläfrigkeit. }\end{array}$ \\
\hline $\begin{array}{l}\text { Kokkelskörner } \\
\text { (Menispermum } \\
\text { cocculus L.) }\end{array}$ & $\begin{array}{l}1 \text { Hund, } \\
1 \text { Katze }\end{array}$ & $\begin{array}{l}\text { taumelnde Körperbewegungen; } \\
\text { Salivation; Erbrechen; tonisch- } \\
\text { klonische, epileptiforme Krampf- }\end{array}$ \\
\hline
\end{tabular}




\begin{tabular}{|c|c|c|}
\hline Gift & Versuchstiere & pathologische Befunde \\
\hline $\begin{array}{l}\text { Brechnu } \beta \\
\text { (Strychnos } \\
\text { nux-vomica L.) }\end{array}$ & 3 Hunde & $\begin{array}{l}\text { anfälle mit Schreien; Aussetzen } \\
\text { von Atmung und Herzaktion. } \\
\text { Sektion: geblähte, hyperämische } \\
\text { Lungen; Dilatation des Herzens; } \\
\text { venöse Einflußstauung; geringe } \\
\text { Rötung der Magenschleimhaut. } \\
\text { generalisierte, tetanische Krampf- } \\
\text { anfälle, ausgeprägter Opisthoto- } \\
\text { nus, Spasmus cynicus; Aussetzen } \\
\text { von Atmung und Herzaktion. } \\
\text { Sektion: Zwerchfellkrämpfe; ge- } \\
\text { blähter Magen; inkonstant ent- } \\
\text { zündliche Rötung der Magen- und } \\
\text { Darmschleimhaut; Dilatation des } \\
\text { Herzens; venöse Einflußstauung. }\end{array}$ \\
\hline $\begin{array}{l}\text { Bittere Mandeln } \\
\text { (Prunus amygdalus } \\
\text { Batsch. var. amara } \\
\text { Focke) }\end{array}$ & $\begin{array}{l}2 \text { Tauben, } \\
1 \text { Storch }\end{array}$ & $\begin{array}{l}\text { Respirationsstörungen; frühzei- } \\
\text { tige Erstickungskrämpfe, Bewußt- } \\
\text { losigkeit. } \\
\text { Sektion: hellrotes und flüssiges Lei- } \\
\text { chenblut; Hirnblutungen; inkon- } \\
\text { stant Rötungen der Schleimhäute } \\
\text { des Verdauungstraktes. }\end{array}$ \\
\hline $\begin{array}{l}\text { Blauer Eisenhut } \\
\text { (Aconitum napellus L.) }\end{array}$ & 1 Hund, 1 Wolf & $\begin{array}{l}\text { Erbrechen; Respirationsstörun- } \\
\text { gen; Lähmungserscheinungen, } \\
\text { später Krämpfe. } \\
\text { Sektion: inkonstant entzündliche } \\
\text { Rötung der Magen- und Darm- } \\
\text { schleimhaut. }\end{array}$ \\
\hline $\begin{array}{l}\text { Weiße Nieswurz } \\
\text { weißer Germer } \\
\text { (Veratrum album L.) }\end{array}$ & 1 Hund & $\begin{array}{l}\text { Erbrechen; Krämpfe. } \\
\text { Sektion: entzündliche Rötung der } \\
\text { Magenschleimhaut. }\end{array}$ \\
\hline $\begin{array}{l}\text { Magisterium Jalapae } \\
\text { (aus Exogonium } \\
\text { purga Bentham) }\end{array}$ & 1 Hund & $\begin{array}{l}\text { taumelnde Körperbewegungen; } \\
\text { Kopfnicken; abführende Wirkung. } \\
\text { Sektion: entzündliche Rötung der } \\
\text { Magen- und Darmschleimhaut. }\end{array}$ \\
\hline
\end{tabular}




\begin{tabular}{|c|c|c|}
\hline Gift & Versuchstiere & pathologische Befunde \\
\hline $\begin{array}{l}\text { Flores antimonii } \\
\text { albi }\left(\mathrm{Sb}_{2} \mathrm{O}_{3}\right)+ \\
\text { Crocus metallorum } \\
\left(\mathrm{Sb}_{2} \mathrm{O}_{3}, \mathrm{KSbO}_{2}, \mathrm{KSbS}_{2}\right)\end{array}$ & 1 Hund & $\begin{array}{l}\text { Erbrechen. } \\
\text { Sektion: geblähter Magen; Rötung } \\
\text { der Magenschleimhaut. }\end{array}$ \\
\hline $\begin{array}{l}\text { Vitrum antimonii } \\
\left(\mathrm{Sb}_{2} \mathrm{O}_{3}, \mathrm{Sb}_{2} \mathrm{~S}_{3}\right)\end{array}$ & 1 Hund & $\begin{array}{l}\text { taumelnde Körperbewegungen; } \\
\text { Erbrechen; Krampfbewegung des } \\
\text { Kopfes. } \\
\text { Sektion: geblähter Magen; ent- } \\
\text { zündliche Rötung der Magen- } \\
\text { schleimhaut. }\end{array}$ \\
\hline $\begin{array}{l}\text { Mercurius sublimatus } \\
\left(\mathrm{HgCl}_{2}\right)\end{array}$ & 1 Hund & $\begin{array}{l}\text { Erbrechen; Diarrhoe. } \\
\text { Sektion: geblähter Magen; ent- } \\
\text { zündliche Rötung der Darm- } \\
\text { schleimhaut; Erosion der Peyer- } \\
\text { schen Plaques. }\end{array}$ \\
\hline $\begin{array}{l}\text { Quecksilberschmier- } \\
\text { kur }+ \text { Mercurius } \\
\text { vivus }(\mathrm{Hg}) \text { p.o. } \\
+ \text { Mercurius subli- } \\
\text { matus }\left(\mathrm{HgCl}_{3}\right) \text { p.o. }\end{array}$ & 1 Hund & $\begin{array}{l}\text { Salivation; Stomatitis, Gingivitis; } \\
\text { Auftreibung des Abdomens; } \\
\text { blutiges Erbrechen; blutige Stühle; } \\
\text { Teerstühle. } \\
\text { Sektion: geblähte, hyperämische } \\
\text { Lungen; Dilatation des Herzens; } \\
\text { venöse Einflußstauung; flüssiges } \\
\text { Leichenblut; blutige Lymphe in } \\
\text { Ductus thoracicus und Cisterna } \\
\text { chyli; Entzündung des Oesopha- } \\
\text { gus-, Magen- und Darmschleim- } \\
\text { haut; gangränöse Abschnitte am } \\
\text { Magendarmkanal; blutiges Extra- } \\
\text { vasat im Bauchraum. }\end{array}$ \\
\hline $\begin{array}{l}\text { Mercurius dulcis } \\
\left(\mathrm{Hg}_{2} \mathrm{Cl}_{2}\right)\end{array}$ & 1 Hund & $\begin{array}{l}\text { Abführwirkung; Exsikkose. } \\
\text { Sektion (unauffälliger Befund am } \\
\text { Magendarmkanal). }\end{array}$ \\
\hline
\end{tabular}




\section{Wepfers Theorie der Giftwirkung}

Auf dem Boden der experimentellen Befunde formulierte Wepfer seine Vorstellungen über die Pathophysiologie der Wasserschierlingsvergiftung und anderer Vergiftungen mit «warmen », « erhitzenden » Giften. Bei seinen Tierversuchen hatte er häufig eine ähnliche Befundkonstellation festgestellt: 1. Symptome von seiten des Gastrointestinaltraktes (Erbrechen, Diarrhoe, Entzündung der Schleimhäute), 2. Symptome des Nervensystems (verschiedene Formen von Krämpfen, «taumelnde» Körperbewegungen). Hinzu kam, daß Wepfer mit der bloßen makroskopischen Betrachtung an Blut und Lymphe meist keine Veränderungen wahrnehmen konnte. Unter diesen Voraussetzungen entwickelte er ein Konzept von einer irritativen Giftwirkung. Zusammengefaßt ergibt sich etwa folgendes Bild: Das Gift des W asserschierlings ist von «warmer» und «scharfer» Qualität. Es wirkt durch seine unsichtbaren Stacheln («spicula», «aculei») auf die Magenschleimhaut («Tunica crustacea») ein, durchdringt diese und reizt die darunter liegende nervöse $W$ andschicht des Magens, die «Tunica nervosa» oder «Membrana nervea ». Diese Irritation, verstärkt durch die ausgelöste Konstriktion von Cardia und Pylorus, setzt sich per consensum auf das gesamte Nervensystem fort. Das dynamische Lebensprinzip des Organismus, der Archeus oder - in Wepfers Diktion - der Praeses systematis nervosi, wird in Aufruhr versetzt. Seine «wütenden»Versuche, das lebensbedrohende Gift abzuwehren, bekunden sich in der Vergiftungssymptomatik, insbesondere in heftigen Krämpfen. Die anderen experimentell untersuchten Pflanzengifte wirken in ganz ähnlicher Weise. ${ }^{17}$

Die beiden wesentlichen Elemente in Wepfers Theorie der Giftwirkung sind: 1. die irritatio per consensum (sive per sympathiam) des Nervensystems durch die primäre Magenreizung, 2. das Postulat eines obersten, vitalen Prinzips des Nervensystems in Gestalt eines personifizierten Praeses systematis nervosi, der die Reizung perzipiert und darauf reagiert. Wepfer hat selbst seinen Praeses systematis nervosi mit dem Archeus Johann Baptist van Helmonts und der Anima sensitiva der Peripatetiker identifiziert. ${ }^{18}$ Grundlegend für die Hypothesen der irritatio per consensum und des Praeses systematis nervosi waren die Ausführungen von Francis Glisson, dem Begründer der Irritabilitätslehre, der in diesem Zusammenhang von Wepfer zitiert wird. ${ }^{19}$ In seinem «Tractatus de ventriculo et intestinis » (1677) nennt Glisson klinische Beispiele für eine «irritatio praeternaturalis per consensum »: Bei einer Darmkolik trete häufig ein Harnverhalt auf, da die Fasern 
des Sphincter vesicae sich per consensum mitkontrahierten. Umgekehrt könne es bei einem Nierenstein und einer Nierenkolik zu Darmbeschwerden kommen. Die Darmkolik und ebenso die Nierenkolik reizten den Magen per consensum zum Erbrechen. Ein Gallenwegsverschluß führe «secundario et per sympathiam » zu kolikartigen Magenschmerzen. ${ }^{20}$ Pathophysiologische Vorstellungen dieser Art waren es, die den Hintergrund zu Wepfers Theorie einer irritativen Giftwirkung bildeten.

Weiterhin waren die geltenden Konzepte des 17. Jahrhunderts über die Darmresorption richtungsweisend. Durch die Entdeckung der mesenterialen Lymphgefäße (Gaspare Aselli, 1622) und des Ductus thoracicus (Jean Pecquet, 1647) war die alte Theorie der venösen Resorption von der einer lymphatischen Darmresorption aller Nahrungsbestandteile verdrängt worden. ${ }^{21}$ So wird eines der Hauptargumente Wepfers für die irritative, sympathische und gegen die resorptive Giftwirkung verständlich: Die Krämpfe träten so kurze Zeit nach der peroralen Giftapplikation auf, daß das Gift noch nicht hätte resorbiert und zum Gehirn und Rückenmark transportiert werden können. Besonders die Vergiftungen mit Kokkelskörnern (Pikrotoxin) und bitteren Mandeln (Blausäure), die Latenzzeiten von nur wenigen Minuten zeigten, waren geeignet, diese Auffassung zu stützen. ${ }^{22}$

Wesentlicher noch als das Problem der Resorptionsgeschwindigkeit war für Wepfers Theorie der Umstand, daß er bei seinen Experimenten durch die makroskopische Inspektion allein in den Därmen der Tiere die Gifte häufig nicht wiederfinden konnte und ihm Blut und Lymphe unverändert erschienen. Wenn also die Gifte überhaupt ins Blut gelangten, folgerte Wepfer, dann nur in so geringer Menge, daß sie wohl kaum auf diesem Wege das Nervensystem zu heftigen Krämpfen reizten. ${ }^{23}$

Die Theorie einer sympathischen Giftwirkung hat sich im Prinzip bis in das 19. Jahrhundert hinein gehalten. Erst mit der weiteren Aufdeckung der venösen Darmresorption und dem chemischen Nachweis der Gifte in den Geweben wurde die Lehre von der sympathischen Wirkungsweise zugunsten der resorptiven Theorie aufgegeben. ${ }^{24}$ Wepfers Überlegungen zu den Wirkungsmechanismen der Gifte sind überwiegend durch vitalistisches Denken (Glisson, van Helmont) geprägt. Wenn er im Schierlingsgift winzige Stacheln vermutet, die in die Magenwand hineinstechen, so werden hier darüber hinaus Korpuskularvorstellungen und iatrophysikalisch-mechanistische Ansätze erkennbar. Von den Iatrochemikern übernimmt Wepfer gelegentlich den Begriff der Fermentation. ${ }^{25}$ Seine Einstellung gegenüber der chemiatrischen Säure-Alkali-Theorie ist allerdings eher zurückhaltend. ${ }^{26}$ Wepfers 
pathophysiologische Vorstellungen, wie sie sich uns in seinem toxikologischen Werk darstellen, basieren somit gleichermaßen auf den Ergebnissen umfangreicher eigener empirischer Forschung wie auch auf führenden Konzepten der theoretischen Medizin des 17. Jahrhunderts.

\section{Anmerkungen}

1 Wepfer, Johann Jakob, Cicutae aquaticae historia et noxae. Commentario illustrata. Basileae, Apud Joh. Rodolphum König. Imprimebat Joh. Rodolphus Genathius. 1679. (Im Folgenden zitiert als: Cicuta.)

Zur Biographie Wepfers siehe: Eichenberger, Pietro, Johann Jakob Wepfer (1620-1695) als klinischer Praktiker, Basel 1969 (Basler Veröffentl. z.Gesch. d.Med.u.d.Biol., Fasc. 26); idem, Autobiographisches von Johann Jakob Wepfer (1620-1695) in einem Briefwechsel mit Johann Conrad Brotbeck (1620-1677), in: Gesnerus 24 (1967) 1-23. Zu Wepfers Abhandlung über den Wasserschierling siehe: Fischer, Hans, Johann Jakob Wepfer 1620-1695. Ein Beitrag zur Medizingeschichte des 17.Jahrhunderts, Zürich 1931; Maehle, Andreas-Holger, Johann Jakob Wepfer (1620-1695) als Toxikologe, Med. Diss. Bonn 1983.

${ }^{2}$ Cicuta, S.5-9.

3 Cicuta, S. 59-68.

4 Vgl. Fischer (Anm. 1), S. 56-58.

5 Boehm, R., Über den giftigen Bestandtheil des Wasserschierlings (Cicuta virosa) und seine Wirkungen; ein Beitrag zur Kenntnis der Krampfgifte, in: Arch.ex. Path. Pharm. 5 (1876) 279-310.

${ }^{6}$ Gessner, Otto, Gift- und Arzneipflanzen von Mitteleuropa, 3. Aufl., hrsg. v. G. Orzechowski, Heidelberg 1974, S. 13-15.

7 Vgl. Ober, William B., Did Socrates die of hemlock poisoning?, in: N. Y. State J. Med.77 (1977) 254-258.

${ }^{8}$ Cicuta, S.34: «Verum utcunque haec evincant Cicutam particulis calidis, acribus et aculeatis, quibus abundat, hos effectus in istis praestitisse, nullum tamen facile assensum impetrabo; obstabunt potissimum Dioscoridis authoritas, a quo caetera turba, quicquid nominis habet in re, mutuata est et in sua transtulit; deinde Socratis mors, plausibile frigus Cicutae concilians. »

${ }^{9}$ Cicuta, S. 17-23.

${ }^{10}$ Cicuta, Praefatio (I-II): «Primum paucae pagellae erant quae de Cicuta Aquatica meditabar: verum postquam convulsiones easque atrocissimas a cicuta prope omnium ore frigida, imprimis aquatica, et congelante excitari in animum inducere non possem, de ejus temperamento dubitare coepi. (...) multivaria versabam animo: tandem rem experimentis peragendam judicavi.»

11 Cicuta, S. 157.

12 Elsholtz, Johann Sigismund, Clysmatica nova, Ed.sec., Coloniae Brandenburgicae 1667, S. 18-19. Zitiert in Cicuta, S. 134: «Minus culpabor si venenorum agendi rationem in brutis explorem, ut hominibus prosim; quod etiam alii nuper fecerunt, inter quos Exc.D. Joh.Sigismund Elsholtz. Serenissimi Electoris Brandenburg. Medicum. Ordinar. nomino, qui Clysmat. Nov.p.18. in venam siphone stagma arsenicale ingessit.» 
Harder, Johann Jakob, De noxis Cicutae terrestris, Ad Virum Celebratissimum Joh. Jacobum Wepferum, Archiatrum Scafusanum. In: Miscellanea curiosa sive Ephemerides medico-physicae Germanicae Academiae Imperialis naturae curiosorum, Dec.II. ann.3. (1684), S. 230-239. Über intravenöse Injektionen S. 232-233.

${ }^{13}$ Buess, Heinrich, Die historischen Grundlagen der intravenösen Injektion, Aarau 1946, S. 109.

${ }^{14}$ Cicuta, Praefatio (VI-V): «Si qua in re (= Vergiftungen) haesitent Philiatri, vel praecipue hac methodo veritatem indagare poterunt idque sine magnis sumptibus, aut anxia sollicitudine: saepe canes, quos Anatomicorum martyres vocant, Anatomae vivae destinant, ut Ventriculi, intestinorum, diaphragmatis, cordis motus, vasa chylifera, lymphatica receptaculum chyli, ductum thoracicum et similia videant; una eademque opera cani ante sectionem aliquid horum (= Gifte) ingerere poterunt, votorum utrinque compotes fient et saepe, quae desiderant, luculentius conspicient: E.gr. fiat periculum Nuce vomica, detur primum illa integra, quam quidam nullas convulsiones efficere ajunt, postea in frustula concisa, aut limata; aut infundatur ejus essentia, vel si spiritus vini experimentum infidum reddere cogitetur, ejus extractum in jusculo solutum ingeratur (...). Exhibeantur haec cani vetulo, mediocris aetatis, aut lactanti, jejuno plenove ventriculo: observatur sedulo numerus, series et vehementia symptomatum: secetur canis vivus, aut statim post mortem; inspiciantur interna gulae, ventriculi intestinorum: incidantur vasa sanguifera majora, dum adhuc calet, occurent certe multa ad majora praelucescentia.»

${ }^{15}$ Cicuta, S. 132: «(...), laudatissima experimentorum certamina Nob.D. Redi et Cl. D. Charras indefesto studio in naturam veneni viperini inquirentium malui imitari (...).»

Siehe: Redi, Francesco, Osservazioni intorno alle vipere, Firenze 1664; Charas, Moyse, Nouvelles expériences sur la vipère ... avec une suite des nouvelles expériences sur la vipère et une dissertation sur son venin, Paris 1672. (Die « Nouvelles expériences sur la vipère » erschienen erstmals Paris 1669.)

${ }^{16}$ Eine Reihe von Wepfers Kollegen aus der Schweizer und süddeutschen Nachbarschaft leisteten mit weiteren Tierversuchen eigene toxikologische Beiträge, die Wepfer in seine Schrift über den Wasserschierling mitaufgenommen hat. Jene sind: Johann Conrad Brunner aus Diessenhofen (Brechnuß), der württembergische Hofarzt Elias Rudolph Camerarius (Kaiserkrone), Christoph Harder aus Schaffhausen (Kokkelskörner), Johann Jakob Harder aus Basel (Erdschierling), Johann Heinrich Hiller aus Stuttgart (Bittere Mandeln), Melchior Hurter aus Schaffhausen (Kokkelskörner, Jalape), Heinrich Screta von Zavorziz aus Schaffhausen zusammen mit Johann Heinrich Hiller aus Stuttgart (Bittere Mandeln), Johann Christoph Simon aus Tübingen (Wasserschierling) und Johann Conrad Steuzel aus Villingen (Wasserschierling). Die Methodik entspricht hier der von Wepfer angewandten.

${ }^{17}$ Cicuta, S.317-318: "Rationibus et experimentis existimo me deprehendisse, Cicutam calidam et acrem esse, suisque aculeis membranam nerveam potissimum ventriculi feriisse, ac fibras ejus circulares, membranae arctissime fibrillis nerveis conjunctas, ad violentam constrictionem irritasse; tum ob vellicationis vehementiam, tum ob constrictionis atrocitatem, maxime circa orificia ventriculi, ubi nervi insigniores apparent, et Praesidem systematis nervosi in furorem actum, unde convulsiones; et cor in consensum raptum fuisse (...). Ut hac de re certius, aut saltem verisimilius aliquid statuere possem aliorum venenorum tum vegetabilium experimenta et observationes, tum Amicorum, tum proprias adjunxi, et quasi in subsidium vocavi, ex quibus didici prope eadem ratione ventriculum afflixisse, ac fere eodem ordine symptomata produxisse.» 
${ }^{18}$ Cicuta, S. 100: «Hunc Praesidem (sc. systematis nervosi) Nob. Helmont. tum in his motibus convulsivis, tum alibi, inserviturum expediendis nodis difficilibus, vocat Archeum (...)». Cicuta, Praefatio (III): «Crebram mentionem facio Praesidis systematis nervosi, quam per me cum Physicis peripateticis Animam sensitivam statuere poteris.»

${ }^{19}$ Cicuta, S.100: «(...) aliquo modo necessario statuendus videtur Praeses aliquis toti Systemati nervoso praefixus, qui quandoque hos tumultus (= Krämpfe) imperet; quemlibet enim motum animalem praecedit perceptio et cognitio aliqua, hanc sequitur appetitus rei gratae aut aversio rei noxiae, a quibus imperatur motus, qui quod imperatum fuit exsequitur: de quibus videatur Exc. D.Glisson. de Percept. et Appetitu Nat. et de Gula, Ventriculo et Intestin. In quibus solide et doctissime hoc argumentum tractat. »

Siehe: Glisson, Francis, Tractatus de natura substantiae energetica, seu de vita naturae, ejusque tribus primis facultatibus, I. perceptiva, II. appetitiva, et III. motiva, naturalibus etc., Londini 1672; idem, Tractatus de ventriculo et intestinis, Amstelodami 1677.

${ }^{20}$ Glisson, Francis, Tractatus de ventriculo et intestinis, Amstelodami 1677, S.198. Ganz ähnliche Beispiele führt Wepfer, Cicuta, S.94-95, an.

${ }^{21}$ Mani, Nikolaus, Darmresorption und Blutbildung im Lichte der experimentellen Physiologie des 17. Jahrhunderts, in: Gesnerus 18 (1961) 85-146; idem, Die historischen Grundlagen der Leberforschung, II. Teil, Basel/Stuttgart 1967, S.84-103.

${ }_{22}^{2}$ Cicuta, S. 187-188, 246-247.

${ }^{23}$ Cicuta, S. 98-99, 156, 168-169, 187-189, 215-216, 220, 246-247.

${ }^{24}$ Earles, M.P., The theory of sympathetic action, in: Pharm.J.177 (1956) 123-124.

${ }^{25}$ Cicuta, S. 24-26.

${ }^{26}$ Cicuta, Praefatio (III): «Alkalium et acidorum pugnam nullam observavi in ventriculo, quam vere talem dicere ausus fuissem. »

\section{Summary}

With his work on the water hemlock from 1679 Johann Jakob Wepfer establishes the method of systematical examination of poisons based on animal experiments. The experimental animals are poisoned per os by various vegetable and mineral substances. The ensuing symptoms are recorded in detail. Later the animals are vivisected in order to study the effects of poisons directly in the interior of the organism. The further postmortem dissection particularly concentrates on the condition of the gastrointestinal mucosa. Symptomatology and postmortem results are correlated and lead Wepfer to the theory of an irritant and sympathetic mode of action of the water hemlock and other poisonous plants: Lesions of the gatrointestinal canal caused by the invisible "spicules" of the poison irritate "per consensum " the Archaeus or Praeses systematis nervosi, i.e. the leading dynamic vital principle of the organism. This irritation per consensum causes convulsions and other symptoms. Influences of vitalistic thought (Glisson, van Helmont) as well as mechanistic approaches are evident in Wepfers theory of toxic effects.

Dr.med. Andreas-Holger Maehle, Institut für Geschichte der Medizin der Universität Göttingen, Nikolausberger Weg 7b, D-3400 Göttingen 